\title{
How rapidly can diastolic function alter with acute afterloading: insights from a closed chest/closed pericardium acute afterload porcine model
}

\author{
Nina Jakuš1, George R. Sutherland², Maja Čikeš1, Peter Haemers'1, Jens-Uwe Voigt', \\ Frank Rademakers', Jan D'hooge', Piet Claus ${ }^{1 *}$ \\ ${ }^{1}$ Catholic University of Leuven, Leuven, Belgium \\ ${ }^{2}$ University Hospital Brussels, Brussels, Belgium
}

Background: The speed and timing of changes in diastolic function parameters during acute LV loading and unloading is unknown.

Methods: In a closed chest, closed pericardium porcine model, LV systolic pressure was increased by $30 \%$ using a transient descending aortic partial balloon inflation. Morphology and mechanical changes were monitored in real time by echocardiography, as were changes in left and right heart diastolic pressures by 3 Millar catheters. Simultaneous blood pool and tissue Doppler data (radial and long axis) were acquired during afterload changes.

Results: In 7 animals, acute loading consistently induced LV dilatation and a rightwards ventricular septal shift due to lateral LV pericardial restraint. This reduced RV volume. Afterload increase caused an immediate decrease in early diastolic filling wave (E) and lateral wall velocity (E'). This was mirrored by an immediate increase in both early diastolic LV (LVDPmin) and LA (LADPmin) pressure (Figure 1). Pulmonary vein flow gradually reduced with decreased late flow. The rightwards septal shift induced a small but significant increase in RVDPmin and an increase in mean RA pressure, but failed to increase pulmonary artery pressure.
Acute afterload release immediately returned $E$ values to baseline, but E' showed a transient further 3 to 5 beat increase before it normalized.

Conclusion: Acute afterloading variably elevates all cardiac diastolic pressures and reduces LV relaxation. These early changes in myocardial mechanics occur simultaneously with changes in LVDPmin. Right heart diastolic pressures increase due to the septal shift which both reduced RV volume and decreased LV compliance but did not elevate pulmonary pressure. E'/E recovery differed which could be attributed to transient preload changes.

KEYWORDS: acute ventricular afterload, pericardial constraint, septal shift, diastolic pressure, pulsed Doppler echocardiography.

\section{Received: $20^{\text {th }}$ Mar 2013}

*Address for correspondence: KU Leuven, Cardiovascular Imaging and Dynamics, UZ Herestraat 49 - box 7003, 3000 Leuven, Belgium.

Phone: $+32-16-3-49017$

E-mail: Piet.Claus@med.kuleuven.be

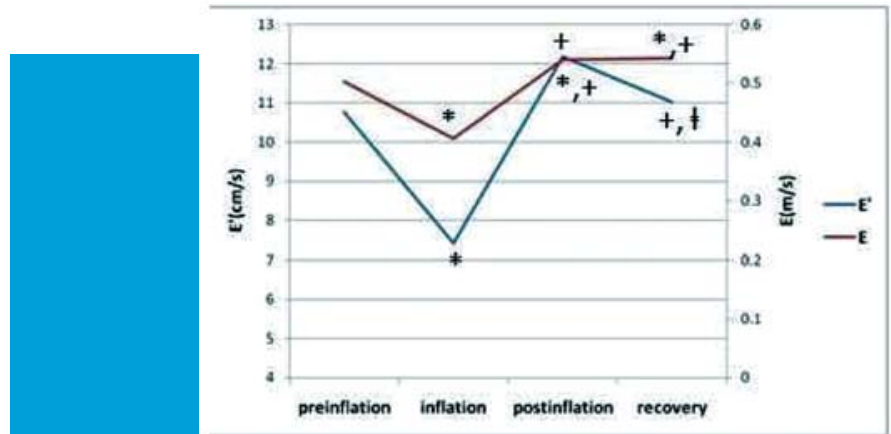

* $(p<.02)$ vs. preinflation $+(p<.01)$ vs.inflation

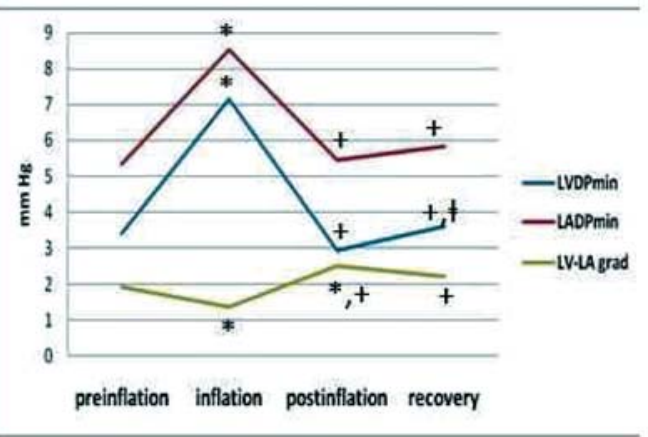

$\neq(\mathrm{p}<.01)$ vs.postinflation

Figure 1. Left panel: Early diastolic filling wave $(E)$ and lateral wall velocity $\left(E^{\prime}\right)$ before, during and after acute pressure increase. Right panel: Left ventricular and atrial early diastolic pressure and transmitral pressure gradient before, during and after acute pressure increase.

\section{Literature}

1. Gillebert TC, Lew WY. Influence of systolic pressure profile on rate of left ventricular pressure fall. Am J Physiol. 1991;261(3 Pt 2):H805-13.

2. Lavine SJ, Lavine JA. The effect of acute hypertension on left ventricular diastolic pressures in a canine model of left ventricular dysfunction with a preserved ejection fraction and elevated left ventricular filling pressures, J Am Soc Echocardiogr. 2006; 19:1350-8.

3. Borlaug BA, Melenovsky V, Redfield MM, Kessler K, Chang HJ. Abraham TP, et al. Impact of arterial load and loading sequence on left ventricular tissue velocities in humans J Am Coll Cardiol. 2007;50:1570-7.

4. Crawford MH, Badke FR, Amon KW. Effect of undisturbed pericardium on left ventricular size and performance during acute volume loading. Am Heart J. 1983;105(2):267-72. 